\title{
Satzglieder, Kasus und semantische Rollen: eine Einführung
}

\section{EVA ENGELS}

Abteilung für Englisch, Institut für Sprache, Literatur und Kultur, Universität Aarhus, Dänemark

\section{STEN VIKNER}

Abteilung für Englisch, Institut für Sprache, Literatur und Kultur, Universität Aarhus, Dänemark

\begin{abstract}
Section 1 introduces the three concepts grammatical function, case and semantic role. Using examples from German and Danish, it is shown in section 2 that although these concepts have certain links to one another, there are no strict correlations between them. Section 3 shows that this is also the case in languages with a much richer case system, such as Finnish.'
\end{abstract}

\section{EINFÜHRUNG}

\subsection{Satzglieder}

Konstituenten eines Satzes unterscheiden sich dahingehend, welche grammatische Funktion (z.B. Subjekt, Objekt, Adverbial etc.) sie in dem Satz übernehmen. Einige dieser Satzgliedfunktionen sind in (1) exemplifiziert:

Dt. a. Hans schenkt dem Kind das Stofftier.

Subjekt $(\mathrm{Su})$

b. Hans schenkt dem Kind das Stofftier.

Direktes Objekt (DO)

c. Hans schenkt dem Kind das Stofftier. Indirektes Objekt (IO)

d. Hans wartet auf seinen Freund. Präpositionalobjekt (PräO)

e. Er kommt jeden Tag. Adverbial (Adv)

f. Der Vortrag ist eine Zeitverschwendung. Subjektsprädikativ (SuPrä) g. Wir finden den Vortrag eine Zeitverschwendung.

Objekts-

prädikativ (OPrä)

Tidsskrift for Sprogforskning • Årgang 4 • Nr. 1-2 • 2006, pp. 17-37 


\subsection{Kasus}

Unter dem Begriff Kasus wollen wir die morphologische Markierung von Substantiven, Adjektiven, Numeralien, Artikeln und Pronomina für unterschiedliche Formen wie z.B. Nominativ, Akkusativ oder Dativ verstehen:

(2) Deutsche Artikel und Substantive

\begin{tabular}{|l|l|l|l|l|}
\hline & Maskulinum & Femininum & Neutrum & Plural \\
\hline NOM & der/ein Tisch & die/eine Mütze & das/ein Brett & $\begin{array}{l}\text { die Tische/ } \\
\text { Mützen/Bretter }\end{array}$ \\
\hline AKK & den/einen Tisch & die/eine Mütze & das/ein Brett & $\begin{array}{l}\text { die Tische/ } \\
\text { Mützen/Bretter }\end{array}$ \\
\hline DAT & dem/einem Tisch & der/einer Mütze & dem/einem Brett & $\begin{array}{l}\text { den Tischen/ } \\
\text { Mützen/Brettern }\end{array}$ \\
\hline GEN & des/eines Tischs & der/einer Mütze & des/eines Bretts & $\begin{array}{l}\text { der Tische/ } \\
\text { Mützen/Bretter }\end{array}$ \\
\hline
\end{tabular}

(3) Deutsche Pronomina

\begin{tabular}{|l|l|l|l|l|}
\hline & Maskulinum & Femininum & Neutrum & Plural \\
\hline NOM & er & sie & es & sie \\
\hline AKK & ihn & sie & es & sie \\
\hline DAT & ihm & ihr & ihm & ihnen \\
\hline GEN & seiner & ihrer & seiner & ihrer \\
\hline
\end{tabular}

Wie die Tabellen (2) und (3) zeigen, verfügt das Deutsche über vier Kasus, die jedoch nicht unbedingt für alle Formen unterschiedlich ausgeprägt sind. Den Zusammenfall verschiedener Kasusformen bezeichnet man als Synkretismus. So weisen etwa Nominativ und Akkusativ im Femininum dieselben Formen auf; dasselbe gilt im Neutrum und im Plural.

Während im Deutschen alle nominalen Konstituenten für Kasus markiert werden, ist in anderen Sprachen wie dem Dänischen oder Englischen morphologischer Kasus nur im Pronominalsystem sichtbar; Substantive dagegen unterliegen keiner morpho-phonologischen Alternation hinsichtlich Kasus. 
Dänische Pronomina

\begin{tabular}{|l|l|l|l|l|l|}
\hline & Maskulinum & Femininum & Utrum & Neutrum & Plural \\
\hline NOM & han & hun & den & det & de \\
\hline AKK & ham & hende & den & det & dem \\
\hline
\end{tabular}

Oben haben wir den Genitiv zu den Kasus des Deutschen gerechnet, aber nicht zu den Kasus des Dänischen. Der Grund dafür ist, dass es im Deutschen Verben und Präpositionen gibt, die ihrem Argument Genitiv zuweisen:

$$
\begin{aligned}
& \text { Dt. a. Die Angehörigen gedachten seiner. } \\
& \text { b. Die Expo } 2000 \text { war trotz des Defizits ein Riesenerfolg. }
\end{aligned}
$$

Gleichfalls konnten in sehr frühen Stadien des Dänischen (hier durch das Altnordische, abgekürzt "AN", repräsentiert) bestimmte Verben und Präpositionen ihrem Argument Genitiv zuweisen. Dies ist aber im heutigen Dänisch nicht mehr möglich:

$$
\begin{aligned}
& \text { AN a. Heraðsmenn leituðu } \underline{\text { hennar. }} \quad \text { (Nygaard 1905: 143) } \\
& \text { Dän. b. *Herredsmoend søgte hendes. } \\
& \text { Bezirksleute suchten ihrer.GEN } \\
& \text { 'Bezirksleute suchten sie.' } \\
& \text { AN a.Hann kømr til konungs atsetu. (Gordon 1957: 27) } \\
& \text { Dän. b. *Han kom til kongens } \underline{\text { boligs. }} \\
& \text { er kam zu des-Königs Wohnsitzes.GEN } \\
& \text { 'Er kam zum Wohnsitz des Königs.' }
\end{aligned}
$$

Formen wie seiner und des Defizits können also im Deutschen sowohl den Verbalkasus Genitiv (5) als auch den Possessiv (8) enkodieren. Entsprechende Ausdrücke im Dänischen dagegen können nur als Possessiv verwendet werden:

(8) Dt. a. Nach seiner Meinung überraschte die Größe des Defizits die Politiker.

Dän. b. Efter hans mening overraskede underskuddets størrelse politikerne. 


\subsection{Semantische Rollen}

Mit dem Begriff semantische Rolle werden die semantischen Relationen der Konstituenten zum Prädikat bezeichnet. So selegiert beispielsweise verfolgen in (10)a,b ein Agens (= den Verfolger) und ein Thema (= den Verfolgten). In der Literatur wurden diverse semantische Rollen vorgeschlagen; einige davon sind unter (9) aufgelistet.

(9) a. Agens führt die Handlung aus.

b. Thema (Patiens) verändert sich durch die Handlung; wird der Handlung unterworfen.

c. Experiencer nimmt etwas wahr, empfindet.

d. Rezipient/Benefaktiv: Empfänger; durch die Handlung Begünstigte(r).

e. Instrument: Mittel, mit dem eine Handlung ausgeführt wird.

f. Ort des Geschehens.

g. Zeit des Geschehens.

Dt. a. Der Detektiv verfolgt den Verdächtigen.

Agens

b. Der Detektiv verfolgt den Verdächtigen.

Thema

b'. Karl hört die Kinder.

Thema

c. Karl hört die Kinder.

Experiencer

d. Maria gibt der Frau die Schlüssel.

Rezipient

e. Hans isst die Suppe mit einem Löffel.

Instrument

f. Susanne spielt im Garten.

Ort

g. Wir wollen jeden Tag Kuchen essen.

Zeit

\section{VERKNÜPFUNGEN ZWISCHEN SATZGLIEDERN, KASUS UND SEMANTISCHEN ROLLEN}

Dieser Abschnitt geht auf die Relationen von Satzglied, Kasus und semantischer Rolle ein. Es soll gezeigt werden, dass zwar keine 1:1-Korrelationen zwischen ihnen bestehen, sie aber auch nicht in einem völlig willkürlichen Zusammenhang zueinander stehen.

\subsection{Verknüpfungen und Ambiguitäten}

Kasusmarkierungen können zur Kennzeichnung der Satzgliedfunktionen sowie der semantischen Rollen von Argumenten dienen. Ein Agens muss in einem Aktivsatz vom Subjekt realisiert werden, und üblicherweise tritt im Deutschen 
das Subjekt im Nominativ auf. ${ }^{3}$ Die Kasusdistinktionen in (11) ermöglichen es somit, über die Satzgliedfunktion zu erschließen, welche der semantischen Rollen von beißen die Argumente jeweils übernehmen: Während die Nominativmarkierung von der Hund in (11)a darauf verweist, dass dieses Substantiv das Agens ausdrückt, deutet der Akkusativ den Hund in (11)b auf dessen semantische Rolle als Thema hin.
Dt. a. Der Hund
beisst
den Postboten.
NOM, Su, Agens
AKK, DO, Thema
'Der Postbote wird vom Hund gebissen.'
b. Den Hund
beisst
der Postbote.
AKK, DO, Thema
NOM, Su, Agens
'Der Hund wird vom Postboten gebissen.'

Wie (12) zeigt, können Kasussynkretismen zu Ambiguitäten führen. Aufgrund der fehlenden morpho-phonologischen Differenzierung ist eine eindeutige Bestimmung der Satzgliedfunktionen sowie der semantischen Rollen der Argumente in diesen Sätzen nicht möglich: Da sowohl die Frau als auch das Pferd für Nominativ oder Akkusativ markiert und somit Subjekt oder Objekt sein könnten, lässt sich nicht entscheiden, welches der Argumente die Rolle des Agens bzw. des Themas übernimmt. Allerdings ist anzumerken, dass es eine starke Tendenz dazu gibt, die satz-initiale Konstituente als Subjekt und somit als Agens zu interpretieren.
Dt. a. Das Pferd
beisst
die Frau.
NOM, Su, Agens
AKK, DO, Thema
'Die Frau wird von dem Pferd gebissen.'
AKK, DO, Thema
$\mathrm{NOM}, \mathrm{Su}$, Agens
'Das Pferd wird von der Frau gebissen.' 
EVA ENGELS UND STEN VIKNER
b. Die Frau
beisst
das Pferd.
$\mathrm{NOM}, \mathrm{Su}$, Agens
AKK, DO, Thema

'Das Pferd wird von der Frau gebissen.'

AKK, DO, Thema

NOM, Su, Agens

'Die Frau wird von dem Pferd gebissen.'

Da im Dänischen Kasusunterschiede bei Substantiven nicht morphophonologisch markiert sind (vgl. Abschnitt 1.2.), sind Sätze mit substantivischem Subjekt und Objekt wie in (13) generell ambig. Wie im Deutschen besteht jedoch auch in dieser Sprache die Präferenz, das satz-initiale Argument als Subjekt aufzufassen. Eine eindeutige Interpretation ist nur mit kasus-eindeutigen Pronomina wie in (14) möglich.
Dän. a. Hunden
bider
postbudet.
NOM, Su, Agens
AKK, DO, Thema
'Der Hund beisst den Postboten.'
AKK, DO, Thema
NOM, Su, Agens
'Den Hund beisst der Postbote.'
b. Postbudet
bider
hunden.
NOM, Su, Agens
AKK, DO, Thema
'Der Postbote beisst den Hund.'
AKK, DO, Thema
NOM, Su, Agens
'Den Postboten beisst der Hund.'

(14)

Dän. a. De

NOM, Su, Agens

'Sie beißen ihn.'

$\begin{array}{lll}\text { b. Dem } & \text { bider } & \text { han. } \\ \text { AKK, DO, Thema } & & \text { NOM, Su, Agens }\end{array}$

bider ham

AKK, DO, Thema

'Sie beisst er.'

$\mathrm{Zu}$ beachten ist, dass diese auf Kasusambiguität beruhende Zweideutigkeit im

Dänischen nur in Hauptsätzen mit nur einem Verb auftritt: In den 
skandinavischen Sprachen ermöglicht die Position des finiten Verbs in Nebensätzen sowie die Position eines nicht-finiten Verbs (in Haupt- und in Nebensätzen), Subjekt und Objekt auseinander zu halten. In diesen Fällen folgt das Verb dem Subjekt, während es einem Objekt vorangeht (soweit dieses sich nicht in satz-initialer Position befindet, vgl. (15)b). Die Sätze in (15) erlauben somit jeweils nur eine einzige Interpretation. Im Deutschen dagegen, wo nichtfinite Verben sowie finite Verben in eingebetteten Sätzen in rechts-peripheren Positionen erscheinen, bleibt die Mehrdeutigkeit bestehen, vgl. (16). Die Wortstellung hilft bei der Interpretation dieser Sätze nur insoweit, als zwei kasusambige Argumente, wie oben bereits erwähnt, vorzugsweise als Subjekt < Objekt aufgefasst werden.
Dän. a. Hunden
har bidt
postbudet.
NOM, Su, Agens
AKK, DO, Thema
'Der Hund hat den Postboten gebissen.'
b. Hunden
har
postbudet
bidt.
AKK, DO, Thema
NOM, Su, Agens

'Den Hund hat der Postbote gebissen.'

$$
\begin{gathered}
\text { c. ... fordi hunden bider postbudet. } \\
\text { NOM, Su, Agens } \\
\text { AKK, DO, Thema } \\
\text {... weil der Hund den Postboten beisst.' }
\end{gathered}
$$

$\begin{array}{clll}\text { Dt. a. Das Pferd } & \text { hat } & \text { die Frau } & \text { gebissen. } \\ \text { NOM, Su, Agens } & \text { AKK, DO, Thema } & \end{array}$

'Die Frau ist von dem Pferd gebissen worden.'

AKK, DO, Thema

NOM, Su, Agens

'Das Pferd ist von der Frau gebissen worden.'

b. ... weil das Pferd die Frau
NOM, Su, Agens AKK, DO, Thema

AKK, DO, Thema NOM, Su, Agens

'... weil das Pferd von der Frau gebissen wird.' 
Die Beispiele in (12), (13) und (16) haben gezeigt, dass ambige Kasusmarkierungen keine klare Zuordnung von Satzgliedfunktionen und semantischen Rollen zulassen. Aber selbst wenn man solche Kasussynkretismen außer Betracht lässt, gibt es keine strikten Korrelationen zwischen Satzgliedern, Kasus und semantischen Rollen. Um nachzuweisen, dass diese drei Eigenschaften nicht direkt miteinander verbunden sind, werden wir zeigen, dass sie unabhängig voneinander variieren können. Die folgenden Abschnitte veranschaulichen dies anhand von Satzpaaren, die sich hinsichtlich einer bzw. zwei dieser drei Eigenschaften unterscheiden (" $\neq$ " in (17)), während sie die andere(n) konstant halten ("=" in (17)). Die Kombinationsmöglichkeiten sind in (17) aufgelistet. Diejenigen Kombinationen, bei denen alle drei Eigenschaften gleich bleiben bzw. alle variieren, werden aus der Diskussion ausgeschlossen, da sie nichts über eine eventuelle Verknüpfung von zwei der drei Eigenschaften aussagen können.

(17) Mögliche Kombinationen

\begin{tabular}{|l|l|l|}
\hline Satzglied & Kasus & semantische Rolle \\
\hline$=$ & $\neq$ & $\neq$ \\
\hline$=$ & $\neq$ & $\neq$ \\
\hline$=$ & $=$ & $=$ \\
\hline$\neq$ & $=$ & $=$ \\
\hline$\neq$ & $=$ & $\neq$ \\
\hline$\neq$ & $\neq$ & $=$ \\
\hline$\neq$ & $\neq$ & $\neq$ \\
\hline
\end{tabular}

Abschnitt

2.2.

2.3.

2.4 .

$--$

2.5 .

2.6.

2.7 .

\subsection{Satzglied $=$, Kasus $\neq$, semantische Rolle $\neq$}

Verschiedene Verben weisen ihren Argumenten unterschiedliche semantische Rollen und Kasus zu. So realisiert etwa das direkte Objekt von vermissen ein Thema im Akkusativ, das von fehlen einen Experiencer im Dativ. 
Beispiel (18) zeigt, dass abhängig vom Verb dasselbe Satzglied verschiedene semantische Rollen ausdrücken und unterschiedliche Kasusformen annehmen kann.

\subsection{Satzglied $=$, Kasus $\neq$, semantische Rolle $=$}

In den Sätzen in (18) variieren sowohl die semantische Rolle als auch der Kasus des Objekts. Es ist aber keinesfalls so, dass bestimmte semantische Rollen an spezifische Kasus gebunden sind. Das direkte Objekt von unterstützen und helfen in (19) trägt die semantische Rolle des Rezipienten, das von vermissen und gedenken in (20) die des Themas. Der Kasus dieser Objekte korreliert nicht mit deren semantischer Rolle: Das Objekt von unterstützen und vermissen steht im Akkusativ, das von helfen im Dativ und das von gedenken im Genitiv.

Dt. a. Sie unterstützen den Mann.

b. Sie helfen dem Mann.
DO, AKK, Rezipient

DO, DAT, Rezipient

DO, AKK, Thema

DO, GEN, Thema

\subsection{Satzglied $=$, Kasus $=$, semantische Rolle $\neq$}

Ähnlich können Argumente mit derselben Satzgliedfunktion und demselben Kasus auch unterschiedliche semantische Rollen enkodieren. Beispielsweise sind die Subjekte in (21) beide für Nominativ markiert, variieren aber hinsichtlich ihrer semantischen Rolle: Das Subjekt von vermissen in (21)a drückt einen Experiencer aus, das von fehlen in (21)b ein Thema. Ebenso können direkte Objekte mit gleichem Kasus unterschiedliche semantische Rollen realisieren: Vermissen selegiert ein Thema als Akkusativobjekt (22)a, beeindrucken einen Experiencer (22)b.

\section{Dt. a. Das Mädchen vermisst den Vater. \\ b. Der Vater fehlt dem Mädchen.}

$\mathrm{Su}, \mathrm{NOM}$, Experiencer

$\mathrm{Su}, \mathrm{NOM}$, Thema 
Die Beispiele in (18)-(22) haben gezeigt, dass Kasus und semantische Rolle nicht miteinander korrelieren. Vielmehr werden sie unabhängig voneinander vom jeweiligen Verb zugewiesen.

\subsection{Satzglied $\neq$, Kasus $=$, semantische Rolle $=$}

In den in den Abschnitten 2.2.-2.4. besprochenen Satzpaaren war die Satzgliedfunktion der Argumente keiner Alternation unterworfen. Unter der Annahme, dass das Objekt eines monotransitiven Verbs immer sein direktes Objekt ist, zeigen die Sätze in (23), dass Argumente mit derselben semantischen Rolle und demselben Kasus verschiedene Satzgliedfunktionen ausüben können: Der Dativ-Rezipient dem Gastgeber ist direktes Objekt von danken in (23)a, aber indirektes Objekt von geben in (23)b.

$$
\begin{aligned}
& \text { Dt. a. Die Frau dankt dem Gastgeber. } \\
& \text { b. Die Frau gibt dem Gastgeber ein Buch. }
\end{aligned}
$$$$
\text { DO, DAT, Rezipient }
$$

IO, DAT, Rezipient

\subsection{Satzglied $\neq$, Kasus $=$, semantische Rolle $\neq$}

Unterschiedliche Satzglieder mit verschiedenen semantischen Rollen können auch mit demselben Kasus markiert werden. Nicht nur Argumente sondern auch Adverbiale können als nominale Konstituenten realisiert werden und müssen dann Kasus aufweisen, vgl. Beispiel (24). ${ }^{4}$ In demselben Satz können somit mehrere Satzglieder mit demselben Kasus auftreten, die aber unterschiedliche semantische Rollen tragen. Außerdem gibt es Verben wie lehren in (25), die mehreren Argumenten denselben Kasus zuweisen.

Dt. a. Sie hat letzten Monat einen Brief geschrieben. b. Sie hat letzten Monat einen Brief geschrieben.
DO, AKK, Thema Adv, AKK, Zeit DO, AKK, Thema b. Er lehrt mich die Lieder Schuberts.
IO, AKK, Rezipient 


\subsection{Satzglied $\neq$, Kasus $\neq$, semantische Rolle $=$}

Auch die letzte Kombination von Satzpaaren belegt, dass eine bestimmte Satzgliedfunktion nicht an eine spezifische Kasusform und semantische Rolle gebunden ist. Argumente mit derselben semantischen Rolle können unterschiedliche Satzgliedfunktionen übernehmen und unterschiedliche Kasus aufweisen. Beispielsweise ähneln sich die Verben vermissen und fehlen darin, dass sie beide sowohl ein Thema als auch einen Experiencer selegieren. Sie unterscheiden sich aber dahingehend, durch welches Satzglied diese semantischen Rollen ausgedrückt und mit welchem Kasus sie markiert werden.

\section{Dt. a. Das Mädchen vermisst den Vater. \\ b. Der Vater fehlt dem Mädchen.}

Dt. a. Das Mädchen vermisst den Vater.

b. Der Vater fehlt dem Mädchen.
DO, AKK, Thema

$\mathrm{Su}, \mathrm{NOM}$, Thema

$\mathrm{Su}, \mathrm{NOM}$, Experiencer

DO, DAT, Experiencer

\subsection{Passiv}

Ein typisches Beispiel für den unter 2.7. besprochenen Fall stellen auch AktivPassiv-Paare dar: Bei Passivierung ändert sich die Satzgliedfunktion und der Kasus eines Arguments unter Erhalt seiner semantischen Rolle. Wie in (28) ersichtlich, wird das direkte Objekt des Aktivsatzes im Passiv zum Subjekt angehoben und mit Nominativ gekennzeichnet; das Subjekt des Aktivsatzes kann durch eine Präpositionalphrase in den Passivsatz integriert werden. Die semantischen Rollen der Argumente verändern sich dabei nicht.

Dt. a. Der Hund beisst den Jungen.

b. Der Junge wird (vom Hund) gebissen.
DO, AKK, Thema

$\mathrm{Su}, \mathrm{NOM}$, Thema

Bei der Passivierung sind zwischensprachliche Kontraste in Hinblick darauf zu beobachten, welche Argumente zum Subjekt promoviert werden können. So kann etwa im Deutschen nur ein Akkusativobjekt im werden-Passiv zum Subjekt angehoben und Nominativ-markiert werden; ein Dativobjekt behält bei der Bildung des werden-Passivs dagegen seinen Kasus bei, siehe Beispiel (29) und (30). Das bekommen-Passiv dagegen promoviert ein zugrundeliegendes Dativ-Objekt zum Subjekt im Nominativ, vgl. (31)d. 
(29) Dt. a. Sie unterstützt $\underline{i h n}$.

AKK

b. Er wird von ihr unterstützt.

NOM

c. *Ihn wird von ihr unterstützt.

AKK

(30) Dt. a. Sie hilft $\underline{\text { hhm }}$.

DAT

b. *Er wird von ihr geholfen.

NOM

c. Ihm wird von ihr geholfen.

(31)

Dt.
a. Der Junge schenkt
dem Mann
den Hund.
$\mathrm{Su}, \mathrm{NOM}$, Agens
IO, DAT, Rez.
DO, AKK, Thema

b. Der Hund wird

dem Mann

geschenkt.

$\mathrm{Su}, \mathrm{NOM}$, Thema

IO, DAT, Rezipient

c. *Der Mann wird

den Hund

geschenkt.

$\mathrm{Su}, \mathrm{NOM}$, Rezipient

DO, AKK, Thema
d. DerMann bekommt den Hund geschenkt.
$\mathrm{Su}, \mathrm{NOM}$, Rezipient $\quad$ DO, AKK, Thema
e. *DerHund bekommt dem Mann geschenkt.
$\mathrm{Su}, \mathrm{NOM}$, Thema IO, DAT, Rezipient

Im Deutschen erlauben also unterschiedliche Hilfsverben die Promotion eines Objekts im Akkusativ bzw. Dativ zum Nominativ-Subjekt (werden- vs. bekommenPassiv). Im Unterschied dazu können im Schwedischen und Norwegischen beide Objekte einer Doppel-Objekt-Konstruktion im Passiv mit demselben Hilfsverb zum Subjekt im Nominativ angehoben werden.
(32) Norw. a. Marie gav
ham
IO, AKK, Rezipient
den.
DO, AKK, Thema

Marie gab ihm es.' 
b. Han

$\mathrm{Su}, \mathrm{NOM}$, Rezipient

'Ihm wurde es gegeben.'

c. Den

$\mathrm{Su}, \mathrm{NOM}$, Thema

'Es wurde ihm gegeben.' ble gitt den.

DO, AKK Thema

ble gitt ham

IO, AKK, Rezipient

Im Englischen und Dänischen dagegen kann zumeist nur das ursprünglich indirekte Objekt im Passiv als Subjekt realisiert werden; d.h. Passivbildung in diesen Sprachen verhält sich spiegelbildlich zum deutschen werden-Passiv, vgl. (33)b,c und (35)b,c gegenüber (31)b,c. Anhebung des Thema-Arguments zum Subjekt ist nur möglich, wenn der Rezipient als Präpositionalphrase realisiert wird, wie Beispiel (34)b und (36)b veranschaulichen.

Eng. a. Mary gave

him

IO, AKK, Rezipient

'Mary gab ihm sie.'

b. $\mathrm{He}$

was given

Su, NOM, Rezipient

'Ihm wurden sie gegeben.'

c. *They

were given

$\mathrm{Su}, \mathrm{NOM}$, Thema

'Sie wurden ihm gegeben.'

(34) Eng. a. Mary gave

them

DO, AKK, Thema

'Mary gab sie ihm.'

b. They

were given

$\mathrm{Su}, \mathrm{NOM}$, Thema

'Sie wurden ihm gegeben.' them.

DO, AKK, Thema

them.

DO, AKK, Thema

him.

IO, AKK, Rezipient to him.

PräO, Rezipient 


\section{EVA ENGELS UND STEN VIKNER}

Dän. a. Marie gav

ham

IO, AKK Rezipient

'Marie gab ihm sie.'

b. Han

blev givet

Su, NOM, Rezipient

'Ihm wurden sie gegeben.'

c. ${ }^{*} D e$

blev givet

$\mathrm{Su}, \mathrm{NOM}$, Thema

'Sie wurden ihm gegeben.'

(36)

Dän. a. Marie gav

dem

DO, AKK, Thema

'Marie gab sie ihm.'

b. De

$\mathrm{Su}, \mathrm{NOM}$, Thema

'Sie wurden ihm gegeben.' dem.

DO, AKK, Thema

dem.

DO, AKK, Thema

ham.

IO, AKK, Rezipient

til ham.

PräO, Rezipient

til ham.

PräO, Rezipient

Noch ein anderes Muster der Passivierung von Doppel-Objekt-Konstruktionen ist im Isländischen zu beobachten, wo zwar das ursprünglich direkte Objekt im Passiv Nominativ zugewiesen bekommt und die Verbalkongruenz steuert, jedoch normalerweise das weiterhin Dativ-markierte indirekte Objekt in der Subjektsposition auftritt; das nominativische Thema-Argument erscheint in post-verbaler Objektsposition, vgl. Abschnitt 2.1.
Isl. a. Hann gaf
öllum kennurum
sama toekifaerið.
er $\quad$ gab
allen Lehrern.DAT
dieselbe Chance.AKK

'Er gab allen Lehrern dieselbe Chance.'

(Holmberg \& Platzack 1995: 208) 


$\begin{array}{lll}\text { Öllum kennurum } & \text { vargefið } & \text { sama toekifarið. } \\ \text { allen Lehrern.DAT } & \text { wurde gegeben } & \text { dieselbe Chance.NOM }\end{array}$

'Allen Lehrern wurde dieselbe Chance gegeben.'

(Gunnar Hrafn Hrafnbjargarson, persönl. Mitt.)

c. *Sama toekifoerið vargefið öllum kennurum.

dieselbe Chance.NOM wurde gegeben allen Lehrern.DAT

'Dieselbe Chance wurde allen Lehrern gegeben.'

\subsection{Zusammenfassung}

Wie in den Abschnitten 2.2.-2.8. dargelegt, bestehen im Deutschen und Dänischen (sowie in anderen Sprachen) keine strikten Korrelationen zwischen Satzgliedfunktion, Kasus und semantischer Rolle. Die Betrachtung des Finnischen im folgenden Kapitel verweist darauf, dass dieser Mangel nicht auf einer zu geringen Ausprägung des Kasussystems in diesen Sprachen beruht.

\section{FINNISCH}

\subsection{Lokalkasus}

Obwohl das Finnische über ein differenziertes Kasussystem verfügt, lässt sich auch in dieser Sprache keine 1:1-Korrelation zwischen Satzglied, Kasus und semantischer Rolle beobachten. So bilden etwa sechs der fünfzehn Kasus ${ }^{5}$ des Finnischen insofern ein einheitliches Teilsystem von Lokalkasus, als sie in ihren Grundbedeutungen Ort und Richtung ausdrücken. Wie mit der Abbildung in (38) und den Beispielen in (39) veranschaulicht, ist die Struktur des Systems zweidimensional und bestimmt sowohl den Ort ('innerhalb' vs. 'außerhalb') als auch die Richtung der Handlung ('am Ort' vs. 'von etwas weg' vs. 'auf etwas hin'), so dass anhand der Kasusmarkierung lokale Relationen im Finnischen eindeutig ausgedrückt werden können. 
(38) Finnische Lokalkasus

\begin{tabular}{|c|c|c|c|}
\hline & & \multicolumn{2}{|c|}{ Ort } \\
\hline & & innerhalb & außerhalb \\
\hline \multirow{3}{*}{ 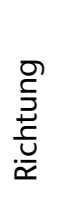 } & am Ort & -ssa (Inessiv, "in") & -lla (Adessiv, "nahe") \\
\hline & weg von & -sta (Elativ, "heraus") & -lta (Ablativ, "von weg") \\
\hline & hin zu & -Vn (Illativ, "hinein") & -lle (Allativ, "zu hin") \\
\hline
\end{tabular}

(Karlsson 2000: 126)

(39) Finn. a. Kaapissa on kahvia.

INESSIV

'Im Schrank ist Kaffee.'

(Lehtinen 1963: 120ff.)

b. Liisa ottaa kahvia kaapista.

'Liisa nimmt Kaffee aus dem Schrank.'

c. Liisa panee kahvia kaappiin.

ILLATIV

'Liisa stellt Kaffee in den Schrank.'

d. Kahvi on pöydällä.

ADESSIV

'Auf dem Tisch steht Kaffee.'

e. Otan kahvia pöydältä.

ABLATIV

'Ich werde den Kaffee vom Tisch nehmen.'

\section{f. Panen kahvia pöydälle'.}

ALLATIV

'Ich werde den Kaffee auf den Tisch stellen.'

Diese Kasusformen werden jedoch nicht nur für Lokalangaben genutzt, sondern können alle auch andere adverbiale Bedeutungen enkodieren. So kann beispielsweise der Inessiv als auch der Illativ bestimmte temporale Bedeutungen ausdrücken und der Adessiv einen Possessor oder ein Instrument bezeichnen, vgl. Beispiel (47)c unten. 


\subsection{Subjekts- und Objektskasus}

Auch die Subjekts- und Objektskasus Partitiv, Nominativ und Akkusativ ${ }^{6}$ sind im Finnischen nicht einer einzigen semantischen Rolle und Satzgliedfunktion zuzuordnen. Die Kasuszuweisung scheint von mehreren Faktoren abzuhängen. Ein Substantiv weist Partitiv auf, wenn

(a) das Substantiv auf eine unbestimmte, nicht näher begrenzte (Teil)Menge referiert, wie es in den Beispielen (40)b, (41)b und (42)b der Fall ist,

(b) das Substantiv das Objekt (bzw. das Subjekt gewisser intransitiver Verben) in einem verneinten Satz ist, wie in (43)b und (44)b,

(c) das Substantiv das Objekt eines Verbs ist, das eine andauernde Handlung bezeichnet bzw. nicht-resultativ ist, wie (45) zeigt, oder

(d) das Substantiv das Objekt eines Psych-Verbs ${ }^{7}$ ist - und zwar dann unabhängig davon, ob die Handlung als abgeschlossen betrachtet wird, vgl. Beispiel (46).

Nominativ und Akkusativ sind Default-Kasus; d.h. sie werden dem Subjekt bzw. Objekt zugewiesen, wenn keine der Bedingungen für Partitiv vorliegt.

(40) Finn. a. Tässä on paperi.

NOM

'Hier ist das Papier.'

(Atkinson 1969: 22)

b. Tässä on paperia.

PART

'Hier ist etwas Papier.'

(41) Finn. a. Lapset leikkivät pihalla.

NOM

'Die Kinder spielten im Garten.'

(Manninen 2003: 165)

b. Lapsia leikki pihalla.

PART

'(Einige) Kinder spielten im Garten.'

(42) Finn. a. Mikko söi kakkun.

AKK

'Mikko aß den ganzen Kuchen.'

(Nelson 1998: 157)

b. Mikko söi kakkua.

PART

'Mikko aß (etwas) von dem Kuchen.' 
(43) Finn. a. Haluan kirjan.

AKK

'Ich will ein/das Buch.'

(Lehtinen 1963: 61)

b. En halua' kirjaa.

PART

'Ich will kein Buch/das Buch nicht.'

(44) Finn. a. Pöydällä on kirja.

NOM

'Auf dem Tisch liegt ein Buch.'

(Lehtinen 1963: 116)

b. Pöydällä ei ole’ kirjaa.

PART

'Auf dem Tisch liegt kein Buch.'

(45) Finn. a. Soili luki lehden.

AKK

'Soili las die Zeitung.'

(Nelson 1998: 157)

b. Soili luki lehteä.

PART

'Soili war dabei die Zeitung zu lesen.'

(46) Finn. a. Pelkäämme häntä.

PART

'Wir fürchten ihn.'

(Atkinson 1969: 67f.)

b. He vihasivat meitä.

PART

'Sie hassten uns.'

(47) Finn. a. Opiskelija tappoi professorin syanidilla.

NOM

'Der Student tötete den Professor mit Zyankali.'

(Manninen 2003: 168)

b. Syanidi tappoi professorin.

NOM

'Zyankali tötete den Professor.'

c. Opiskelija tappoi professorin syanidilla.

ADESSIV

'Der Student tötete den Professor mit Zyankali.' 
Die von diversen Faktoren abhängigen Alternationen zwischen Partitiv und Nominativ/Akkusativ verweisen darauf, dass zum einen ein und dasselbe Satzglied (Subjekt bzw. Objekt) unterschiedliche Kasusformen (Partitiv vs. Nominativ/Akkusativ) annehmen kann (vgl. z.B. (41) und (42) oben) und zum anderen verschiedene Satzglieder mit demselben Kasus markiert werden können: Sowohl Subjekt als auch Objekt können im Partitiv erscheinen (siehe z.B. (41)b und (42)b). Des weiteren kann ein Satzglied verschiedene semantische Rollen ausdrücken - und zwar unabhängig von seinem Kasus: Das Subjekt im Nominativ oder Partitiv in (40) ist Thema, das in (41) Agens; das NominativSubjekt in (47)a realisiert ein Agens, das in (47)b ein Instrument. Die semantische Rolle Instrument kann aber ebenso gut auch als Adverbialangabe im Adessiv wie in (47)c ausgedrückt werden. Diese wenigen Beispiele verdeutlichen bereits, dass Satzglied, Kasus und semantische Rolle auch im Finnischen nicht aneinander gebunden sind.

\section{ZUSAMMENFASSUNG}

Als Einführung in die Thematik 'Satzglieder, Kasus und semantische Rollen' haben wir an Beispielen veranschaulicht, was wir unter diesen drei Begriffen verstehen. Kasus, Satzglied und semantische Rolle stehen weder in einer willkürlichen Beziehung zueinander, noch korrelieren sie strikt miteinander. Dies gilt nicht nur für Sprachen wie dem Dänischen oder Deutschen mit keiner bzw. wenig Kasusmarkierung sondern auch für das Finnische, das ein viel ausgeprägteres Kasussystem aufweist.

\section{LITERATUR}

Atkinson, John (1969), A Finnish Grammar, Helsinki: The Finnish Literature Society.

Blake, Barry (1994), Case, Cambridge: Cambridge University Press.

Gordon, Eric V. (1957), An Introduction to Old Norse, 2. Auflage, revidiert durch Arnold R. Taylor, Oxford: Clarendon Press.

Helbig, Gerhard, Joachim Buscha (1986), Deutsche Grammatik. Ein Handbuch für den Ausländerunterricht, Leipzig: Verlag Enzyklopädie.

Hellan, Lars (1988), "The Phrasal Nature of Double Object Clusters", Working Papers in Scandinavian Syntax, 42 (1988), pp. 3-24.

Holmberg, Anders (1986), Word Order and Syntactic Features in the Scandinavian Languages and English, Ph.D., University of Stockholm. 
Holmberg, Anders, Christer Platzack (1995), The Role of Inflection in Scandinavian Syntax, New York: Oxford University Press.

Hrafnbjargarson, Gunnar Hrafn (2004), Oblique Subjects and Stylistic fronting in the History of Scandinavian and English: The Role of IP-Spec, Ph.D., University of Aarhus. [http://hum.uit.no/a/hrafnbjargarson/phd/ghrafn_phd.pdf]

Hrafnbjargarson, Gunnar Hrafn (2005), "Review of The Syntax of Ditransitives: Evidence from Clitics by Elena Anagnostopoulou", Journal of Germanic Linguistics, 17 (2005), pp. 49-63.

Karlsson, Fred (2000), Finnische Grammatik, Hamburg: Buske Verlag.

Lehtinen, Meri (1964), Basic Course in Finnish, The Hague: Mouton.

Manninen, Satu (2003), Small Phrase Layers. A Study of Finnish Manner Adverbials, Amsterdam: John Benjamins.

Nelson, Diane (1998), "Case Competition in Finnish", Nordic Journal of Linguistics, 21 (1998), pp. 145-178.

Nygaard, Marius (1905), Norrøn Syntax, Kristiania: Aschehoug.

Wöllstein-Leisten, Angelika, Axel Heilmann, Peter Stepan, Sten Vikner (1997), Deutsche Satzstruktur. Grundlagen der syntaktischen Analyse, Tübingen: Stauffenburg.

\section{ANMERKUNGEN}

1 Für hilfreiche Kommentare und Kritik danken wir den Teilnehmern an dem 6 . Linguistischen Forschungskolloquium 'Kasus, Satzglied und semantische Rolle' an der Staats- und Universitätsbibliothek Aarhus sowie Per Bærentzen und Carl Vikner.

2 Utrum (dän. foelleskøn) ist das Resultat des Zusammenfalls von Maskulinum und Femininum der Substantive und [-menschlich] Pronomina. Die Bezeichnung ist eine Rückbildung aus Neutrum, eigentlich ne-utrum "keiner von beiden".

3 Ein Nominativ-Subjekt braucht jedoch nicht unbedingt in einem deutschen Satz erscheinen. Es ist umstritten, ob die Sätze unten kein Subjekt oder ein Subjekt im Akkusativ bzw. Dativ aufweisen, siehe Hrafnbjargarson (2004) und die Literaturhinweise dort.

$\begin{array}{lll}\text { Dt. } & \text { a. } \text { Mich friert. } & \text { AKK } \\ & \text { b. } \text { Mir ist kalt. } & \text { DAT }\end{array}$

4 Es gibt nicht nur Akkusativ-markierte Adverbiale wie in (24) sondern auch Genitivmarkierte:

Dt. a. Er besuchte uns eines Abends.

b. Der Kunde verließ den Laden unverrichteter Dinge.

(Helbig \& Buscha 1986: 292)

5 Neben den sechs Lokalkasus und den vier Subjekts- und Objektskasus kennt das Finnische noch den Essiv, Translativ, Abessiv, Komitativ und Instruktiv, die anscheinend keiner besonderen Spezialisierung unterliegen.

6 "Der Akkusativ ist eigentlich keine konkrete Kasusform, sondern eine zusammenfassende Bezeichnung für diejenigen Kasus (Nominativ, Genitiv, -t Akkusativ), die als Objektskasus im Gegensatz zum Partitiv stehen." (Karlsson 2000: 110)

7 Psych-Verben sind Verben, die einen psychischen Zustand oder Prozess bezeichnen. Sie werden auch Experiencer-Verben genannt, da eines ihrer Argumente (nicht 
SATZGLIEDER, KASUS UND SEMANTISCHE ROLLEN: EINE EINFÜHRUNG

notwendigerweise das Subjekt) einen Experiencer ausdrückt und sie kein Agens selegieren. 